\author{
Einführung in das Schwerpunktthema
}

\title{
Ökologischer Strukturwandel: Oldie but Goldie?
}

\section{$\mathrm{V}$} Von Jan Nill und Annette Volkens on einem ökologischen Strukturwandel (ÖSW) ist derzeit kaum noch die Rede. In früheren Jahren einte das Konzept die kritische ökologische Forschung, Umweltverbände und der Umweltproblematik aufgeschlossene Parteien. Heute bestimmen hingegen vornehmlich Begriffe wie ökologische Modernisierung, Öko-Effizienz und Nachhaltigkeit den politischen und wissenschaftlichen Diskurs. Heißt dies, dass der ökologische Strukturwandel auch jenseits der Rhetorik ein Konzept ,,von gestern" ist? Mit den zugrunde liegenden ökologischen Herausforderungen ließe sich eine solche Sichtweise sicherlich nicht begründen. Auch bestreitet kaum jemand, dass eine Wirtschaft und Gesellschaft, die diesen Herausforderungen gerecht werden möchte, im Ergebnis durch andere Strukturen gekennzeichnet sein müsste. Wird Nachhaltigkeit ernst genommen, bleibt ein Prozess des ökologischen Strukturwandels auf der Agenda. Doch der Weg dahin ist beschwerlich, und das schlägt sich zusammen mit veränderten Sichtweisen auch in Begriffsverschiebungen nieder.

\section{Ein antiquiertes Konzept?}

Wissenschaftlich ist das Konzept ökologischer Strukturwandel eng mit den so genannten ,Wendeszenarien“ verknüpft - von den Energiewendeszenarien des Öko-Instituts Anfang der 80-er Jahre bis zu Konzepten der Verkehrs-, Chemieund Agrarwende (1). Die Wendeszenarien betonten die Krisenhaftigkeit des Status Quo sowie die daraus resultierende Notwendigkeit einer Umkehr der Entwicklungsrichtung.

Ursprünglich aus den 80-er Jahren datiert auch eine vor allem auf den Politologen Martin Jänicke zurückgehende einflussreiche begriffliche Unterscheidung zwischen Modernisierung und Strukturwandel. Als ökologische Modernisierung bezeichnet er nur das Segment möglicher Umweltverbesserungen, die durch technischen Fortschritt jenseits von End-ofpipe-Ansätzen zu erzielen sind. Davon grenzt er ökologischen Strukturwandel deutlich ab, mit dem für ihn nicht-technische Lösungen, nämlich eine veränderte Nachfrage- und Infrastruktur und industrieller Strukturwandel verbunden sind (2).
Im Verlauf der 90-er Jahre verändert sich der wissenschaftliche Diskurs. Aus dem stärker werdenden umweltökonomischen Einfluss ergibt sich ein Fokus auf politische Instrumente, der Charakter der damit verbundenen Wirkungen tritt in den Hintergrund. Und das mit von Weizsäcker und Porter verbundene Öko-Effizienz-Konzept betont ökologisch-ökonomische Win-win-Potenziale für alle Akteure. Schließlich beziehen neuere soziologische Ansätze etwa von de Mol ökologische Modernisierung breiter auf Gesellschafts- und nicht nur Technikprozesse, und schließen darin auch Strukturveränderungen ein (3).

\section{Die Zukunft des Konzepts}

Dennoch ist ein Verzicht auf das ÖSW-Konzept wissenschaftlich problematisch (4). Beispielsweise lassen sich beim Öko-Effizienz-Konzept die zur Erreichung der Umweltziele formulierten anspruchsvollen Effizienzsteigerungen - bis hin zu ,Faktor $10 “$ - kaum ohne Strukturveränderungen erreichen. Im Sichtbarmachen dieses Bedarfs, und dem damit verbundenen Einschluss von Veränderungen von Konsumverhalten und Lebensstil liegt eine Ratio von Jänickes Fassung des Strukturwandels. Problematisch ist jedoch seine strikte Trennung zwischen Technik und Struktur. Hierauf deuten nicht nur manche der skizzierten Verschiebungen, sondern auch Erkenntnisse der neueren Innovationstheorie. Diese verweist auf die enge Verbindung zwischen technischen und organisatorisch-sozialen Innovationen, insbesondere wenn es um nicht-inkrementelle Veränderungen geht, die auch Strukturen beeinflussen (5)

Dies verdeutlicht den möglichen wissenschaftlichen Ort eines reformulierten ÖSW-Konzepts: nämlich die Kennzeichnung der Besonderheiten nicht-inkrementeller technischer, ökonomischer, gesellschaftlicher und/ oder politischer Veränderungen, die zu einer Umweltentlastung beitragen. Dies ist nicht notwendig als Gegensatz zu ökologischer Modernisierung zu sehen. Vielmehr schärft das ÖSW-Konzept eine im Modernisierungsbegriff etwas verwischte Dimension, auf die es bei der inhaltlichen Gestaltung entsprechender Politiken durchaus ankommt, denn sie ist schwerer durch- zusetzen. Im Prozess dieser Strukturveränderungen geht es um das geeignete dynamische Zusammenspiel von technischen, institutionellen und sozialen Faktoren (6). Gerade letzteres wird durch das Strukturwandelskonzept besser ausgedrückt als durch den Begriff Wende, der auf eine bipolare Abkehr vom Bisherigen im Sinne einer Reversibilität verweist.

\section{Politisch unattraktiv?}

Im politischen Alltagsdiskurs jenseits von Parteigrundsatzprogrammen hat sich inzwischen jedoch das Modernisierungskonzept weitgehend durchgesetzt. Am augenscheinlichsten ist dies in der rot-grünen Koalitionsvereinbarung, die ausdrücklich ein Programm der ,,ökologischen Modernisierung" formuliert. Wenn es politisch tatsächlich um Strukturwandel geht, wird daher entweder weiter von Modernisierung geredet oder auf die Wende-Begrifflichkeit zurückgegriffen, die derzeit im Agrar- und Energiebereich ein gewisses Revival erlebt.

Augenscheinlich verspricht man sich davon bei der Umsetzung in reale Politik Vorteile. Modernisierung - aber in Krisenzeiten auch Wende, die dann offenbar auch die Thematisierung von Konflikten erlaubt - sind im deutschen Sprachgebrauch positiv im Sinne von Fortschritt besetzt, während Strukturwandel für viele BürgerInnen aber auch die Wirtschaft beängstigend und bedrohlich wirken mag. Manches spricht also dafür, dass sich über das erstere Begriffspaar den Akteuren besser vermitteln lässt, um was es für die Förderung eines ÖSW geht - und so die notwendige Akzeptanz dafür erhöht wird. Dies reflektiert auch die sinkenden Gestaltungsmöglichkeiten nationaler Politik.

Solange dabei der inhaltliche Kern zumindest als Potenzial erhalten bleibt, spricht daher gerade im Hinblick auf die Förderung der Umsetzung eines ÖSW pragmatisch viel dafür, sich politisch nicht zu lange an der Semantik aufzuhalten, sondern sich auf die Substanz der jeweiligen politischen Konzepte im Sinne eines ÖSW zu konzentrieren. Viele politische Entscheidungen kennen Gewinner und Verlierer. Den Verlierern müssen Alternativen angeboten werden - und die müssen wissenschaftlich fundiert und politisch tragbar sein.

Vor diesem Hintergrund beschäftigt sich der vorliegende Schwerpunkt mit den Möglichkeiten und Grenzen einer praktischen Umsetzung eines ökologischen Strukturwandels. Er widmet sich vor allem den Akteuren, die den Prozess in Richtung eines ÖSW befördern können, ihrem möglichen Zusammenspiel sowie übergreifenden Konzepten 
dafür. Anders als üblich klappert er nicht einfach die einzelnen Handlungsfelder Energie (7), Verkehr, Industrie, Landwirtschaft etc. ab, sondern erschließt auch die Querverbindungen und Besonderheiten etwaiger Akteurskonstellationen und deren gegenseitige Beeinflussung. Dabei liegt ein Schwerpunkt auf Praxisaspekten. Dies findet auch darin seinen Ausdruck, dass nicht nur WissenschaftlerInnen, sondern auch Praxisakteure zu Wort kommen. Gestaltet werden die Beiträge überwiegend von Mitgliedern der $A r$ beitsgruppe Ökologischer Strukturwandel der Vereinigung für ökologische Wirtschaftsforschung, Die AG ÖSW ist ein Netzwerk aus vorwiegend jungen HochschulabsolventInnen und Berufstätigen in Unternehmen und gesellschaftlichen Organisationen, Ministerien und Parlamenten, Universitäten und freien Forschungsinstituten, die mit unterschiedlichen Aktivitäten die Ökologisierung von Wirtschaft und Gesellschaft unterstïtzen.

\section{- Die Beiträge im Überblick}

Die Beiträge teilen sich grob in drei Blöcke. Der erste Abschnitt beschäftigt sich mit Handlungsfeld übergreifenden politischen Instrumenten und Konzepten. Mario Meinecke und Bettina Schrader zeigen mit einer Kombination von Zertifikatehandel und Fortsetzung der Ökosteuer eine konkrete Perspektive auf, wie ein ökologischer Strukturwandel mit Hilfe umweltökonomischer Instrumente gefördert werden kann. Darauf folgend geben Klaus Jacob, Jan Nill und Ulrich Petschow einen theoretisch hinterlegten und mit praktischen Beispielen untermauerten Überblick über Strategien, die die mit einem Strukturwandel verbundenen und politisch problematischen Strukturbrüche adressieren.

Der folgende Block fokussiert anhand dreier Beispiele auf einzelne wichtige Akteure des Strukturwandels. Den Einstieg gibt Annette Volkens über den Akteur Staat, wobei Politikintegration und die dafür notwendigen institutionellen Veränderungen - aktuell im Rahmen von Nachhaltigkeitsstrategien - im Zentrum stehen. Daran anschließend betrachtet Wiebke Lass den Akteur KonsumentIn und skizziert kommunikationsorientierte Strategien, wie VerbraucherInnen stärker zu einem Strukturwandel beitragen können. Andrew Murphy stellt dann aus der Sicht eines Praktikers des ethisch-ökologischen Investments die (steigenden) Einflussmöglichkeiten von Finanzdienstleistern und Investoren dar.
Den dritten Teil des Schwerpunktes bilden ausgewählte Handlungsfelder. Veränderungsnotwendigkeit und Probleme im zentralen Feld Verkehr werden von Gerrit Schrammen vor allem anhand von Beispielen für die Freizeitmobilität abgebildet. Den Abschluss macht Marcus Franken, der sich mit notwendigen Reformen des Bildungssektors und ihren Akteuren als Grundlage für einen Strukturwandel beschäftigt.

$\mathrm{Zu}$ guter Letzt ist noch ein Wort zum Entstehungsprozess dieses Schwerpunkts angebracht, an dem noch mehr Akteure beteiligt waren. Die einzelnen Beiträge wurden entsprechend der diskursiven Tradition der AG ÖSW von einzelnen Mitgliedern ausgiebig kommentiert, was entscheidend zum ihrem Erscheinungsbild beitrug. Besonderer Dank gebührt daher neben den AutorInnen Stephan Breuer, Guido Bünstorf, Uwe Büsgen, Kai Schlegelmilch, Uwe Schneidewind und Karsten Schomaker.

\section{Anmerkungen}

(1) Für einen aktuellen Rückblick vgl. die Beiträge von $M$. Hesse, H. Glauber und U. Schneidewind zur AG Wendeszenarien in IÖW (Hrsg.): Regulative Ideen nachhaltigen Wirtschaftens, Berlin 2000, S. 19-22.

(2) Jänicke, M.: Karriere eines Konzepts. Chancen und Grenzen von ökologischer Modernisierung. In: Ökologisches Wirtschaften Nr. 6/00, S. 27-28.

(3) Mol, A.P.J./Spaargaren, G.: Ecological Modernisation Theory in Debate: A Review, in: Mol, A.P.J./ Sonnenfeld, D.A. (Hrsg.): Ecological Modernisation Around the World, London, Portland 2000, S. 17-49.

(4) Vgl. Z.B. Diekmann, J.: Zwischen Effizienz und Suffizienz. In: Ökologisches Wirtschaften, Nr. 3/1999, S.

25-26.

(5) Für einen aktuellen Überblick vgl. Konrad, W./ Nill, J.: Innovationen für Nachhaltigkeit. IÖW-Schriftenreihe 157/01, Berlin 2001.

(6) Vgl. VÖW (Hrsg.): Ökologische Modernisierung. VÖWSchriftenreihe, Berlin 2001 sowie den zusammenfassenden Artikel in Ökologisches Wirtschaften Nr. 1/2001, S. 8-9.

(7) Vgl. hierzu das Spezial „Energiewende in Sicht" der letzten Ausgabe Nr. 3-4/2001 dieser Zeitschrift.

\section{Die Autorlnnen}

Jan Nill, wissenschaftlicher Mitarbeiter am IÖW, und Annette Volkens, Pressesprecherin der VÖW, koordinieren derzeit die VÖW-AG Ökologischer Strukturwandel.

Kontakt: VÖW, Potsdamer Str. 105, 10785 Berlin. Tel. 030/ 8851800, Fax 030/ 8825439,

E-mail: oesw@voew.de, jan.nill@ioew.de, annette. volkens@voew.de

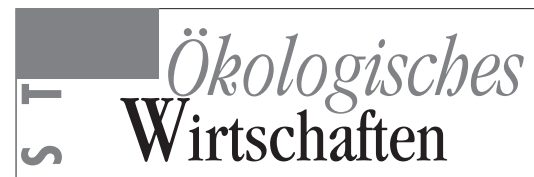

Folgende Ausgaben von Ökologisches Wirtschaften können Sie noch bestellen:
2/98 Global Governance und Umweltpolitik (DM 16,80)
>3-4/98 Perspektiven des Öko-Audit- Systems (inkl. Spezial „Ökologisch- Ökonomische Komplexität“ DM 18,90)
>5/98 Nachhaltige Regional- entwicklung (DM 16,80)
> 6/98 Die Ökonomie der Nach- haltigkeit (DM 16,80)
1/99 Umweltschutz und Unter- nehmen im globalen Wettbewerb (DM 16,80)
- 2/99 Umwelt und Innovation (DM 16,80)
>3/99 Öko-Effizienz (DM 16,80)
-4/99 Europäische Harmonisierung (DM 16,80)

>5-6/99 Städtische Nutzung und Verkehr (inkl. Spezial ,Jahrtausendwende - Zeitenwechsel“ DM 18,90)

>1/00 Konsum zwischen Ethik und Erlebnis (DM 16,80)

-2/00 Sozial-ökologische Unternehmensbewertung (DM 16,80)

3-4/00 Nachhaltige Land- und Ernährungswirtschaft (inkl. Spezial „Greening the Universities“ DM 18,90)

\$5/00 Öko-effiziente

Dienstleistungen (DM 16,80)

-6/00 Integrierte Produktpolitik (DM 16,80)

-1/01 Unternehmen und Nachhaltigkeit (DM 16,80)

- 2/01 Neue Ansätze der Ökonomik (DM 16,80)

3-4/01 Produkt-Ökobilanzen in der Praxis (inkl. Spezial „Energiewende

> in Sicht?"DM 18,90)

Bestellen bel: pan adress

Semmelweißstr. 8

82152 Planegg

Tel: 089/85709-145,

Fax: 089/85709-131 
(c) 20I0 Authors; licensee IÖW and oekom verlag. This is an article distributed under the terms of the Creative Commons Attribution Non-Commercial No Derivates License (http://creativecommons.org/licenses/by-nc-nd/3.o/), which permits unrestricted use, distribution, and reproduction in any medium, provided the original work is properly cited. 